IOS Press

\title{
Erratum
}

\section{Cysteine Supplementation May be Beneficial in a Subgroup of Mitochondrial Translation Deficiencies}

Marina Bartsakoulia, Juliane S. Müller, Aurora Gomez-Duran, Patrick Yu-Wai-Man, Veronika Boczonadi and Rita Horvath

[Journal of Neuromuscular Diseases 3(3), 2016, 363-379, DOI: 10.3233/JND-160178]

http://content.iospress.com/articles/journal-of-neuromuscular-diseases/jnd160178

On page 363, in the author listing, the fourth author was incorrectly listed as Patrick Yu Wai Man. The author's correct name is Patrick Yu-Wai-Man 